\title{
COVID-19 and Dental Environment
}

\author{
Sruthi Lingasamy ${ }^{1}$, Vinej Somaraj ${ }^{2}$ \\ ${ }^{1}$ Senior House Surgeon, Government Coimbatore Medical College and Hospital, Trichy Road, Coimbatore, \\ Tamil Nadu - 641018 \\ ${ }^{2}$ Associate Professor, Department of Public Health Dentistry, Rajas Dental College \& Hospital, Tirunelveli, \\ Tamil Nadu, India - 627105 \\ Corresponding Author: Vinej Somaraj
}

DOI: https://doi.org/10.52403/ijhsr.20220214

\begin{abstract}
The advent of the severe acute respiratory syndrome coronavirus 2 (SARS-CoV-2) and the coronavirus disease it causes has engulfed the entire world, causing serious health concerns. This is a zoonotic infection, similar to other coronavirus infections that are thought to have started in bats and pangolins before being passed on to people. This coronavirus multiplies rapidly once inside the human body. This coronavirus is abundantly present in nasopharyngeal and salivary secretions of afflicted patients once inside the human body, and its dissemination is assumed to be primarily through respiratory droplet/contact. Dental professionals are most likely to encounter patients with SARS-CoV-2 illness, and they will need to be cautious not just in providing care but also in preventing nosocomial infection spread. In addition, special suggestions for patient screening, infection control techniques, and patient care procedures are made for the dentistry office.
\end{abstract}

Keywords: Air Disinfection, Aerosol, COVID-19, Dentistry, Occupational Hazards, SARS-CoV-2

\section{INTRODUCTION}

The new coronavirus variety Novel Coronavirus 2019 is a mutated, extremely contagious form, and the sickness is known as COVID -19. In late 2019, the first cases of a lung ailment with an unknown cause were confirmed in Wuhan, China. The Chinese Centre for Disease Control and Prevention stated on January 8, 2020, that a unique COVID was the causal microorganism of COVID-19. The World Health Organization declared the outbreak to be a public health emergency of worldwide concern on January 30, 2020. ${ }^{1,2}$ Dentists are one of the most vulnerable groups for coronavirus transmission and infection via aerosols. Asymptomatic patients (carriers), as well as those who have an acute respiratory infection, may seek dental care. While treating patients who require urgent or emergency dental procedures is crucial, the primary focus should be to prevent infection from spreading to patients and dental healthcare professionals.

Dentists currently only do emergency dental treatments, during which they must adhere to cross-infection control guidelines. In addition to infection management, COVID-19 has several components that are relevant to dental practice, such as prevention and therapy.

Pre-dental treatment: Patient triage, identification of likely suspects, postponement of non-urgent dental care, administration of dental appointments, and active screening of dental staff are some of the protective practices that should be addressed before the patient enters the dental clinic.

At the dental office: Active patient screening, management of social distance in the dental office, offering sanitation measures to patients, use of facemasks by 
everyone entering the dental office, patient education, use of PPE by the dental team, and management of the dental operating room are just a few of the procedures that must be performed in dental offices.

During dental treatment: During dental procedures, good hand hygiene, providing patients with a preoperative antimicrobial mouth rinse, using rubber dams, highvolume saliva ejectors, and extraoral radiographs, using 4-handed dentistry, avoiding aerosol-generating procedures, one-visit treatment, and environmental cleaning and disinfection procedures should all be used.

Post dental treatment: Following dental treatment, it's important to think about cleaning and sanitising reusable facial protection equipment, as well as managing laundry and medical waste. ${ }^{3,4}$

\section{Possible risk of transmission of COVID- 19 in Dentistry}

The respiratory system's main mechanisms of transmission include droplet transmission and transmission through fomites, which occur often during sneezing, dry coughing, and even talking. The virus has also been observed to be transmitted through the eyes. In light of the COVID-19 disease's primary mode of transmission, dental treatments that result in spit particles being sprayed into the air may increase the risk of infection. Knowing which dental treatments produce virus-carrying aerosols is critical for determining the level of risk that these procedures provide and whether personal protective equipment (PPE) is necessary. As a result, anything that emerges from the patient should be regarded as potentially dangerous. ${ }^{5,6}$ Because the majority of dental equipment are constructed of metal and polymers, the COVID-19 could attach to and linger on these surfaces for several days, posing a danger of virus transmission if not properly cleaned. Not only could the professionals act as transmitters, but they could also become infected during human-to-human transmission via non-invasive salivary secretions such as a patient's cough or sneeze, or treatment procedures such as using a high-speed handpiece or ultrasonic instruments that release aerosols containing saliva or blood bacteria and viruses into the environment. Given that the dissemination of saliva and dental fluids has the potential for viral transmission due to the close distance between patients and professionals, wearing adequate protective clothing is crucial. ${ }^{5,6}$

In a dental practice, PPE and a hand hygiene system should be taken seriously at all times. Because dentists have such close contact with patients and their hands are frequently exposed to oral fluids and aerosols, applying an antiseptic solution before each patient's treatment is critical. Because of their non-toxic nature, ethanolic solutions are ideal for this process. Dental workers must use masks with holes less than 50 microns. These particles, on the other hand, maybe pass through the eyes; thus, wearing suitable goggles or face shields may reduce the risk of infection. To yet, the ADA and CDC only recommend using peroxide to kill the virus. Furthermore, mouthwashes containing $0.2 \%$ chlorhexidine (CHX), $1 \%$ povidone-iodine (P.I.), $1.5 \%$ hydrogen peroxide (H2O2), or $0.05 \%$ hypochlorous acid have been recommended by public health authorities (HOCl). Because saliva flow might potentially replace the virus, $\mathrm{CHX}$ is weak in terms of virucidal capabilities, while the other three (P.I., H2O2, $\mathrm{HOCl}$ ) have excellent virucidal properties but low substantivity. In terms of virucidal and taste properties, $1.5 \%$ hydrogen peroxide is the most clinically acceptable.

\section{Treatment}

COVID-19 has yet to receive an authoritative treatment. Various antiviral drugs, as well as other medication groups, have been used in the past, with varying degrees of efficacy. Azithromycin is a macrolide antibiotic used extensively in dentistry. It is useful in the treatment of children's respiratory infections. For the 
treatment of community-acquired pneumonia, hospitalised patients commonly get the IV form of the medication. Azithromycin is effective against the Zika and Ebola viruses, and when given to patients with a viral illness, it can avoid severe respiratory tract infections. For appropriate cases, other antibiotics such as amoxicillin or clindamycin (in penicillinallergic patients) should be considered if no contraindications exist. Patients with a history of pseudomembranous colitis or ulcerative colitis, for example, are ineligible for clindamycin. ${ }^{4}$ Since the 1930s, chloroquine has been predominantly utilized as an antiparasitic and antimalarial medication. During the present COVID-19 pandemic, numerous countries have claimed that it will be used in their trials to eradicate the illness. Because hydroxychloroquine is a chloroquine derivative with considerably higher solubility and lower toxicity, it is expected to have fewer side effects. It became necessary for dental patients to rely on supportive therapy such as analgesics and non-steroidal anti-inflammatory medications (NSAIDs) to manage their discomfort. The backbone of treatment for mild to moderate COVID-19 instances is supportive analgesic and antipyretic medication. Paracetamol is recommended as the first-line treatment, with ibuprofen as a second-line treatment, according to the WHO. ${ }^{4}$ Unless there is a contraindication, paracetamol can be used as a first-line analgesic; however, if it is ineffective, they can prescribe ibuprofen or other NSAIDs. As a result, wearing disposable waterproof scrubs and bonnets, gloves, eyewear protection, face shields, disposable shoe covers, and masks is highly suggested. ${ }^{5,6}$

The Filtering Face-piece (FFP) mask, which may also block virus particles, is the most commonly recommended PPE for airway protection in dentistry. FFP masks are designed to protect the wearer and are classified into three categories based on their filtration efficiency against powders with a diameter of less than $0.3 \mathrm{~mm}$ : FFP1 (80\% minimal total filtration efficiency),
FFP2 (94 \% minimal total filtration efficiency), and FFP3 (100\% minimal total filtration efficiency) (99\% minimal total filtration efficiency). ${ }^{5}$ The most efficient masks are FFP2/N95, FFP3/N99, and N100, because air droplet COVID-19 particles are predicted to be $0.060 .14 \mathrm{~mm}$ in diameter. Facepieces can be classified as valved or non-valved respirators, in addition to their filtering efficiency. Valved respirators allow for easier air exhalation, resulting in less moisture build-up inside the mask; as a result, they can filter the inhaled air but not the exhaled air. Nonvalved respirators filter both entrance and outflow of air, providing strong two-way protection. ${ }^{1,2}$

Safety glasses Eye protection has always been recommended in the dental industry to prevent mechanical contact with the eyes. agents that are chemical or biological. ${ }^{1,2}$ The ocular pathway is one of the most common ways for Sars-CoV-21 to infect people. Eyewear with enveloping frames and wide lenses should be worn to cover as much of the face as feasible. Plastic shields, on the other hand, maybe preferable to glasses due to their higher ability to protect the face from aerosol droplets. ${ }^{7,8}$

\section{Mechanisms to prevent the spread of COVID-19 in the dental environment}

Updated local guidelines urged avoiding dental services throughout the pandemic outbreak, save for those with an emergency. Each dental professional must be aware of the Sars-CoV-2 transmission channels and perform all necessary procedures in a way that prevents the virus from spreading. ${ }^{3}$ Patients infected with COVID-19 who have a body temperature of more than $37.5^{\circ} \mathrm{C}\left(99.5^{\circ} \mathrm{F}\right)$ should be confined to their homes or hospitalised if they have severe symptoms. ${ }^{4,5}$ For the initial evaluation of patients, a triage space is required, and this area should be set up so that close interaction between individual patients and patients and healthcare workers is minimised. A preliminary examination of patients includes taking their temperature and doing a quick survey to look for signs 
of fever, respiratory problems, cough, or dyspnea in the previous 14 days, as well as contact with others who may have been infected. When surfaces were cleaned with $62 \%, 71 \%$ ethanol, $0.5 \%$ hydrogen peroxide, or $0.1 \%$ sodium hypochlorite, the coronavirus could be effectively eradicated in 1 minute. Sars-CoV-2 appeared to be more stable on plastic and stainless steel than on cardboard or copper; the time until Sars-CoV-2 became inactive was as follows: 72 hours for plastic, 48 hours for stainless steel, 24 hours for cardboard, and 4 hours for copper. ${ }^{9}$

Rinsing before dental operations is an effective way to minimise the bacteria burden in the mouth cavity. Hand hygiene is regarded as the most significant preventive practice for minimising the danger of pathogen transmission between dentists and patients. Soaps and cleansers must be rubbed vigorously on both hands until a thick froth appears. The lipid sheath around the viruses has been demonstrated to break down in this foam, causing viral molecules to disperse and decompose. At concentrations greater than 60\%-65\%, alcohol can dissolve fatty molecules of the external lipid layer of the virus, which leads to disruption of the virus particle; therefore, friction with an alcoholic hand sanitiser is suggested after handwashing. Orthopantomography (OPG) or cone-beam computed tomography (CBCT) are preferred. Because periapical X-rays might produce hypersalivation, coughing, and vomiting, they should be avoided. When using handpieces or ultrasonic equipment, a rubber dam is recommended because it considerably minimises the amount of saliva and/or blood aerosol, resulting in a $70 \%$ reduction in droplets around the surgical field. High-speed saliva ejectors or surgical ejectors can significantly reduce droplet spread during dental treatments, and their use is highly advised. It may also be beneficial to assemble two ejectors at the same time. Dental handpieces can draw and then eject biological fluids and pollutants, which can then be deposited on the patient or the dentist, resulting in cross-infection. Because anti-retraction hand-pieces have been found to successfully prevent bacteria and virus backflow into the tubing system, using hand-pieces without an anti-retraction system should be avoided during the COVID19 pandemic. ${ }^{10,11}$

A few options have been proven to be effective in reducing bacterial and virus loads in dental offices. In the waiting room, safe spacing between patients must be maintained. The majority of Sars-CoV-2 droplets precipitate and settle on surfaces when no aerosol is produced. When handpieces or ultrasonic devices are used, the resulting aerosol can spread the virus into the air, where it can live for up to 3 hours. Ozone is a natural gas that is one of the most effective disinfection techniques for the environment. It generates extremely reactive free radicals that can oxidise bacteria, viruses, organic and inorganic substances, impairing bactericidal activity. Germicidal ultraviolet (UV) radiation is also a viable method for sterilisation: UV radiation can damage microbial DNA and RNA, inhibiting microbe proliferation and lowering infectious organisms' damaging effects. These UV lights can be used in water and air circulation systems with a filtration mechanism to remove powders, germs, and viruses. ${ }^{1,2}$

\section{CONCLUSION}

Dentists are the one among the healthcare professionals who are at constant risk of cross-infection so the protocols for infection control and disinfection have to be maintained with utmost priority. Whatever be the situation, one must be always prepared for anything as the pandemic has not come to an end, even with the inoculation vaccines it will take several years to bring the pandemic to an end.

Funding: None

Ethical Approval: Not Applicable 
Conflicts Of Interest: Authors declares there are no actual or potential conflicts of interest

\section{REFERENCES}

1. Checchi V, Bellini P, Bencivenni D, Consolo U. COVID-19 dentistry-related aspects: a literature overview. Int Dent J. 2020.

2. Barabari P, Moharamzadeh K. Novel Coronavirus (COVID-19) and Dentistry-A Comprehensive Review of Literature. Dent J. 2020;8(2):E53.

3. Spagnuolo G, De Vito D, Rengo S, Tatullo M. COVID-19 Outbreak: An Overview on Dentistry. Int J Environ Res Public Health. 2020;17(6):E2094.

4. Odeh ND, Babkair H, Abu-Hammad S, Borzangy S, Abu-Hammad A, AbuHammad O. COVID-19: Present and Future Challenges for Dental Practice. Int $\mathbf{J}$ Environ Res Public Health. 2020;17(9): E3151.

5. Villani FA, Aiuto R, Paglia L, Re D. COVID-19 and Dentistry: Prevention in Dental Practice, a Literature Review. Int J Environ Res Public Health. 2020 Jun 26;17(12):E4609.

6. Banakar M, Bagheri Lankarani K, Jafarpour D, Moayedi S, Banakar MH, MohammadSadeghi A. COVID-19 transmission risk and protective protocols in dentistry: a systematic review. BMC Oral Health. 2020 Oct 8;20(1):275.

7. Amato A, Caggiano M, Amato M, Moccia G, Capunzo M, De Caro F. Infection Control in Dental Practice During the COVID-19 Pandemic. Int J Environ Res Public Health. 2020;17(13):E4769.

8. Villani FA, Aiuto R, Paglia L, Re D. COVID-19 and Dentistry: Prevention in Dental Practice, a Literature Review. Int $\mathbf{J}$ Environ Res Public Health. 2020;17(12): E4609.

9. Tysiąc-Miśta M, Dubiel A, Brzoza K, Burek M, Pałkiewicz K. Air disinfection procedures in the dental office during the COVID-19 pandemic. Med Pr. 2021;72(1): 39-48.

10. Gurgel BC de V, Borges SB, Borges REA, Calderon PDS. COVID-19: Perspectives for the management of dental care and education. J Appl Oral Sci Rev. 2020;28: e20200358.

11. Odeh N-D, Babkair H, Abu-Hammad S, Borzangy S, Abu-Hammad A, AbuHammad O. COVID-19: Present and Future Challenges for Dental Practice. Int $\mathrm{J}$ Environ Res Public Health. 2020;17(9): E3151.

How to cite this article: Lingasamy S, Somaraj V. COVID-19 and dental environment. Int $J$ Health Sci Res. 2022; 12(2): 105-109. DOI: https://doi.org/10.52403/ijhsr.20220214 